\title{
A Class of Convex Preferences Without Concave Representation*
}

\author{
Paulo Klinger Monteiro ${ }^{\dagger}$
}

\author{
Contents: 1. Introduction; 2. Basic Definitions and Auxiliary Results; 3. Main Theorem; \\ Keywords: Concave Representation, Concavifiable Preferences, Linear Utility. \\ JEL Code: D01, D12.
}

I show that continuous convex preference relations that have affine indifference curves do not have a concave representation if there are two indifference curves that are not parallel. In other words a preference relation with affine indifference curves that has a concave representation has a linear utility representation.

Eu demonstro que uma relação de preferências contínua e convexa que tem curvas de indiferença afins não tem representação côncava se existirem pelo menos duas curvas de indiferença não paralelas. Em outras palavras, uma relação de preferências com curvas de indiferença afins que tem representação côncava tem na verdade uma representação linear.

\section{INTRODUCTION}

Every continuous convex preference relation on $\mathbb{R}_{+}^{l}$ has a continuous utility representation $U$ : $\mathbb{R}_{+}^{l} \rightarrow \mathbb{R}$ which is quasi-concave. It is natural to ask if the preference relation has another representation: one that is concave. The first study on this subject ${ }^{1}$ is de Finetti (1949). There he already mentions the importance of this question in utility theory. Fenchel (1953) has a deeper study of this problem. He presents necessary and sufficient conditions. His conditions are easy to check except one (i. e. condition VII on page 124-125.) Kannai (1977) deepens the study of Fenchel's condition VII - and obtains a formula (see for example, Theorem 2.4 page 9) for the concave representation when there is one.

A nice example of a preference relation that has not a concave representation appears in Arrow and Enthoven (1961) on footnote 6 page 781. The function $U(x, y)=x-1+\sqrt{(1-x)^{2}+4(x+y)}$ is quasi-concave, strictly monotonic. The indifference curve $U^{-1}(u)$ is the straight line connecting $(u / 2,0)$ and $\left(0, \frac{u^{2}+2 u}{4}\right)$. They mention that Fenchel (1953) has proved that such a function cannot have a concave representation. It is not clear however if they mean smooth representations or the general

\footnotetext{
${ }^{*}$ I acknowledge the help of H. Pioner and B. fux Svaiter in getting some old papers. The comments from participants at my talk at Brisbane University is gratefully acknowledged. I acknowledge the financial support of CNPq.

${ }^{\dagger}$ EPGE/FGV, Escola de Pós-Graduação em Economia, Fundação Getulio Vargas, Rio de Janeiro, Brasil. E-mail: paulo.klinger@ fgv.br

${ }^{1}$ A paper with no references!
} 
case. It is easy to check that such a function has no smooth concave representation. Another example is given in Schummer (1998). Analytically his example is $U(x, y)=\frac{2 x}{2-y}, 0<x, y \leq 1$. The indifference curve is the part of the straight line connecting $(u, 0)$ and $(0,2)$ that intersect $(0,1]^{2}$. The example in Schummer has the same spirit ${ }^{2}$ as the example in Aumann (1975) Figure 8 on page 629. Aumann refers to Fenchel (1956) as a source of the example. ${ }^{3}$ So what type of quasi-concave utility function has no concave representation? It is clear from the above examples that it helps if the indifference curves are linear. Here I will prove a strong result in this set up. I show that if a strictly monotonic preference on $\mathbb{R}_{+}^{l}$ has affine indifference curves ${ }^{4}$ and two of these affine sets are not parallel then there is no concave representation. In other words, a preference relation with affine indifference curves that has a concave representation necessarily has a linear utility representation. That is, there exists $b \in \mathbb{R}_{++}^{l}$ such that $U(x)=b \cdot x$ represents the preference relation.

\section{BASIC DEFINITIONS AND AUXILIARY RESULTS}

Let $X \subset \mathbb{R}_{+}^{l}$ be a convex set. A function $U: X \rightarrow \mathbb{R}$ is quasi-concave if $\{x \in X ; U(x) \geq u\}$ is convex for every $u$. It is concave if $U(r x+(1-r) y) \geq r U(x)+(1-r) U(y)$ for every $x, y \in X$ and $0<r<1$. It is easy to check that every concave function is quasi-concave as well.

Definition 1 [concavifiability]. The quasi-concave function $U$ has a concave representation (or is concavifiable) if there is a strictly increasing function $f: U(X) \rightarrow \mathbb{R}$ such that $f \circ U$ is a concave function.

Let $U: X \rightarrow \mathbb{R}$ be quasi-concave and continuous. Let $M:=-\mathbb{R}_{+}^{l}$.

Definition 2. For each $v \in M$ and $u \in U(X)$ define

$$
h(u, v)=\sup \{x \cdot v ; U(x) \geq u\} .
$$

Note that $h(u, v) \leq 0$ since $X \subset \mathbb{R}_{+}^{l}$. The next lemma is a simplified version of (Fenchel, 1953, page $123 \S 53)$.

Lemma 1 [Fenchel]. Suppose $f: U(X) \rightarrow I$ is strictly increasing and continuous onto $I$. Let $g:=f^{-1}$ : $I \rightarrow U(X)$ be its inverse. If $f \circ U$ is concave then $\theta(z):=h(g(z), v)$ is concave for every $v \in M$.

Proof. Let $z^{\prime}, z^{\prime \prime} \in I$ and $0<r<1$. For a given $\epsilon>0$ there exist $x^{\prime}, x^{\prime \prime} \in X$ such that

$$
\begin{gathered}
h\left(g\left(z^{\prime}\right), v\right)-\epsilon<x^{\prime} \cdot v, U\left(x^{\prime}\right) \geq g\left(z^{\prime}\right), \\
h\left(g\left(z^{\prime \prime}\right), v\right)-\epsilon<x^{\prime \prime} \cdot v, U\left(x^{\prime \prime}\right) \geq g\left(z^{\prime \prime}\right) .
\end{gathered}
$$

Therefore if $s=1-r$,

$$
f\left(U\left(r x^{\prime}+s x^{\prime \prime}\right)\right) \geq r f\left(U\left(x^{\prime}\right)\right)+s f\left(U\left(x^{\prime \prime}\right)\right) \geq r z^{\prime}+s z^{\prime \prime}
$$

and thus $U\left(r x^{\prime}+s z^{\prime \prime} \geq g\left(r z^{\prime}+s z^{\prime \prime}\right)\right.$. Hence

$$
h\left(g\left(r z^{\prime}+s z^{\prime \prime}\right), v\right) \geq\left(r x^{\prime}+s x^{\prime \prime}\right) \cdot v \geq h\left(g\left(z^{\prime}\right), v\right)+h\left(g\left(z^{\prime \prime}\right), v\right)-2 \epsilon .
$$

\footnotetext{
${ }^{2}$ The indifferences curves intersect a fixed point in Schummer and Aumann's example.

${ }^{3}$ Presumably the example on III page 501, namely $\phi=x_{2} / x_{1}$.

${ }^{4}$ I. e. translations of hyperplanes.
} 
Since $\epsilon$ is arbitrary the proof is finished.

Definition 3. A set $A \subset \mathbb{R}^{l}$ is affine if it is the translation of a vector subspace. That is $A=a+V$ with $V$ a vector subspace of $\mathbb{R}^{l}$. The affine set $A$ has codimension 1 if $V$ has codimension 1.

Definition 4. The utility function $U: X \rightarrow \mathbb{R}$ has affine indifference curves iffor every $u \in U(X)$ we have that $U^{-1}(u)=H(u) \cap X$ where the affine set $H(u)$ has codimension 1.

The following lemma is central.

Lemma 2. Let $I$ and $J \subset(0, \infty)$ be open intervals. Suppose $g: I \rightarrow J$ and $\phi: J \rightarrow(0, \infty)$ are strictly increasing functions and that $g(I)=J$. Suppose also that for every $k>0$ the function $\min \{g(z), k \phi(g(z))\}$ is convex. Then $g$ and $g \circ \phi$ are convex and there is a $t>0$ such that $\phi(u)=t u$ for every $u \in J$.

The proof is in the Appendix.

\section{MAIN THEOREM}

I begin defining in more detail the class of functions I use.

Let $b(u) \in \mathbb{R}^{l} \backslash\{0\}$ and $\tau(u)>0$ be functions defined for $u \in(0, \infty)$. The set

$$
H(u)=\left\{x \in \mathbb{R}^{l} ; b(u) \cdot x=\tau(u)\right\}
$$

is an affine set with codimension 1 . Dividing by $\tau(u)$ we may suppose without loss of generality that $\tau(u) \equiv 1$ for $u>0$. And therefore

$$
H(u)=\left\{x \in \mathbb{R}^{l} ; b(u) \cdot x=1\right\} .
$$

Lemma 3. Suppose $U: \mathbb{R}_{+}^{l} \rightarrow \mathbb{R}$ is a strictly monotonic function such that $U(0)=0$. Suppose also that $U^{-1}(u) \neq \emptyset$ is affine for every $u>0$. Let $U^{-1}(u)=H(u) \cap \mathbb{R}_{+}^{l}$ where $H(u)$ is defined in (*). Then $b_{i}(u)$ is strictly decreasing for $u>0$, continuous and onto $(0, \infty)$. Moreover $U$ is quasi-concave and continuous.

The proof is in the Appendix.

The theorem I want to prove is the following:

Theorem 1. Suppose $U: \mathbb{R}_{+}^{l} \rightarrow \mathbb{R}_{+}$is a onto strictly monotonic utility function with affine indifference curves. If it has a concave representation then there is a strictly positive vector $b>>0$ such that $V(x)=b \cdot x$ is a representation of $U$.

Proof. Define $\phi_{i}(u)=1 / b_{i}(u)$ for $u>0$. Thus $\phi_{i}$ is continuous and strictly increasing onto $(0, \infty)$. Let $v=\left(-k_{1}, \ldots,-k_{l}\right)<<0$. Then

$$
\max \left\{-\sum_{i} x_{i} k_{i} ; U(x) \geq u\right\}=\max \left\{-\sum_{i} x_{i} k_{i} ; U(x)=u\right\}
$$


The linearity of $U^{-1}(u)$ implies that

$$
\begin{aligned}
& \max \left\{-\sum_{i} x_{i} k_{i}, \sum_{i=1}^{l} \frac{x_{i}}{\phi_{i}(u)}=1\right\} \\
& =-\min \left\{\sum_{i} x_{i} k_{i}, \sum_{i=1}^{l} \frac{x_{i}}{\phi_{i}(u)}=1\right\}=-\min _{i} k_{i} \phi_{i}(u) .
\end{aligned}
$$

Fenchel's Lemma implies the concavity of $-\min _{i} k_{i} \phi_{i}(g(z))$. And therefore $\min _{i} k_{i} \phi_{i}(g(z))$ is convex. Fix $k_{1}=1$ and $j \neq 1$. Making $k_{i} \rightarrow \infty$ for every $i \neq j$ we conclude that

$$
\min \left\{\phi_{1}(g(z)), k_{j} \phi_{j}(g(z))\right\}
$$

is convex for every $k_{j}>0$. Defining $\tilde{g}=\phi_{1} \circ g$ we have that

$$
\min \left\{\tilde{g}(z), k_{j} \phi_{j} \circ \phi_{1}^{-1}(\tilde{g}(z))\right\}
$$

is convex for every $k_{j}>0$. Lemma 2 implies that $\phi_{j} \circ \phi_{1}^{-1}(u)=t_{j} u$ for some $t_{j}>0$. Thus $\phi_{j}(u)=t_{j} \phi_{1}(u)$ since $\phi_{1}\left(\mathbb{R}_{++}\right)=\mathbb{R}_{++}$. Therefore

$$
U^{-1}(u)=\left\{x>>0 ; \sum_{i=1}^{l} \frac{x_{i}}{\phi_{i}(u)}=1\right\}=\left\{x>>0 ; \sum_{i=1}^{l} \frac{x_{i}}{t_{i}}=\phi_{1}(u)\right\}
$$

and therefore $\phi_{1}^{-1}(U(x))=\sum_{i=1}^{l} \frac{x_{i}}{t_{i}}$ is a linear function.

QED

Remark 1. It is easy to generalize $e^{5}$ Theorem 1 to convex subsets with non-empty interior: For each interior point there is a small copy of the positive cone. Then from the theorem the utility is linear in this neighborhood. Then connectedness implies that the linear function is the same everywhere.

Remark 2. The restriction $X \subset \mathbb{R}_{+}^{l}$ is what makes the theorem 1 non-trivial. If $X=\mathbb{R}^{l}$ then the existence of a concave representation would imply that the asymptotic cone of the level sets $\{x ; U(x) \geq u\}$ is constant in $u$. And this by itself would ensure that the indifference curves, $U^{-1}(u)$, are parallel.

\section{BIBLIOGRAPHY}

Arrow, K. \& Enthoven, A. (1961). Quasi-concave programming. Econometrica, 29(4):779-800.

Aumann, R. J. (1975). Values of markets with a continuum of traders. Econometrica, 43:611-646.

de Finetti, B. (1949). Sulle stratificazioni convesse. Annali de Matematica Pura ed Aplicata, XXX(4):173183.

Fenchel, W. (1953). Convex cones, sets and functions. Mimeo, Princeton University.

Fenchel, W. (1956). über konvexe funktionen mit vorgeschriebenen niveaumannigfaltigkeiten. Mathematische Zeitschrift, 63:496-506.

Kannai, Y. (1977). Concavifiability and constructions of concave utility functions. Journal of Mathematical Economics, 4:1-56.

\footnotetext{
${ }^{5}$ I thank Rabee Tourky for mentioning this extension to me.
} 
Saks, S. (1964). Theory of the Integral. Dover Publications, Inc., New York.

Sard, A. (1958). Images of critical sets. Annals of Mathematics, 68(2):247-259.

Schummer, J. (1998). A pedagogical example of non-concavifiable preferences. Unpublished.

\section{APPENDIX}

\section{PROOFS OMITTED IN THE TEXT}

Proof of Lemma 2: Making $k \rightarrow \infty$ we have that $g(\cdot)$ is convex. Dividing by $k$ and making $k \rightarrow 0$ we conclude that $\phi \circ g(\cdot)$ is convex as well. Thus $g$ and $\phi \circ g$ are continuous on $I$ and differentiable except on a countable set. Moreover since $g$ is strictly increasing and convex $g^{\prime}(z)>0$ if it is defined. Thus $g^{-1}$ is differentiable except on a countable set and thus $\phi=(\phi \circ g) \circ g^{-1}$ is differentiable except on a countable set as well. Let $\tilde{I}$ be the set of $i \in I$ such that $g^{\prime}(i)>0$ and $(\phi \circ g)^{\prime}(i)>0$. Thus $I \backslash \tilde{I}$ is a countable set. Suppose now that $\phi^{\prime}(u)>\phi(u) / u$ on a uncountable subset of $J$. Thus there exists an $u^{0}=g\left(z^{0}\right)$ such that

$$
\phi^{\prime}\left(u^{0}\right)>\frac{\phi\left(u^{0}\right)}{u^{0}} \text { and } g^{\prime}\left(z^{0}\right)>0 .
$$

Let $k$ be such that $u^{0}=k \phi\left(u^{0}\right)$. Since

$$
\begin{aligned}
\left.\frac{d}{d z}(g(z)-k \phi(g(z)))\right|_{z=z^{0}} & =g^{\prime}\left(z^{0}\right)-k \phi^{\prime}\left(u^{0}\right) g^{\prime}\left(z^{0}\right)= \\
k g^{\prime}\left(z^{0}\right)\left(\frac{\phi\left(u^{0}\right)}{u^{0}}-\phi^{\prime}\left(u^{0}\right)\right) & <0
\end{aligned}
$$

we have that

$$
\begin{cases}g(z)>k \phi(g(z)) & \text { if } z<z^{0} \\ g(z)<k \phi(g(z)) & \text { if } z>z^{0}\end{cases}
$$

Thus the convexity of $\min \{g(z), k \phi(g(z))\}$ implies that $k(\phi \circ g)^{\prime}\left(z^{0}\right) \leq g^{\prime}\left(z^{0}\right)$. Thus

$$
g^{\prime}\left(z^{0}\right)=k \frac{\phi\left(u^{0}\right)}{u^{0}} g^{\prime}\left(z^{0}\right)<k \phi^{\prime}\left(u^{0}\right) g^{\prime}\left(z^{0}\right) \leq g^{\prime}\left(z^{0}\right)
$$

a contradiction. Now if $\phi^{\prime}(u)<\phi(u) / u$ on an uncountable set we obtain a contradiction by an analogous reasoning. Let $z(u)=\log (\phi(u))-\log (u)$. Thus $z(u)$ is a continuous function such that $z^{\prime}(u)=0$ except on a countable set. Theorem 7.9 page 206 of Saks (1964) implies that $z(u)$ is constant. Then if this constant is written as $\log (t)$ we conclude that $\phi(u)=t u$. This ends the proof.

Remark 3. To prove the last step of the proof above we may use Sard (1958). If $A$ is the set of points where the derivative of $z(u)$ exists and is null then the Corollary on page 254 of Sard (1958) implies that the range is null. Since $A^{c}$ is countable we have a continuous function with a null range. But the only null interval is the one point interval.

Proof of Lemma 3: First note that strict monotonicity implies $b(u)>>0$. To see this suppose $b_{i}(u)>$ $0>b_{j}(u)$. If $U\left(x^{0}\right)=u$ then $x=x^{0}-b_{j}(u) e_{j}+b_{i}(u) e_{i}>>x^{0}$ and $U(x)=u$ a contradiction. Take $t>0$ a real number. And let $u=U\left(t e_{i}\right)$. Then since $t e_{i} \in H(u)$ it follows that $b_{i}(u) t=1$. Thus $b_{i}\left(\mathbb{R}_{++}\right)=\mathbb{R}_{++}$. From $b_{i}\left(U\left(t e_{i}\right)\right)=1 / t$ it follows that $b_{i}$ is strictly decreasing. A decreasing 
function whose range is an interval is continuous. Let me prove the quasi-concavity. Suppose $U(x) \geq u$ and $U(y) \geq u$. If $0<r<1$ we have that $b(u) \cdot x \geq b(U(x)) \cdot x=1$ and $b(u) \cdot y \geq 1$. Then

$$
b(u) \cdot(r x+(1-r) y)=r b(u) \cdot x+(1-r) b(u) \cdot y \geq 1
$$

implies that $U(r x+(1-r) y) \geq u$. I omit the proof of continuity (which is not difficulty). 